\title{
Zum Verhältnis von Theorie und Praxis im Fremdsprachenunterricht
}

\section{On the relation of theory and practice in foreign-language instruction}

\begin{abstract}
Theoretical glottodidactic discussions are not only limited to the analysis and elucidation of the processes of teaching and learning foreign languages. They also create specific concepts for practical implementation. The essence of these processes is interpreted in many scholarly disciplines - for example, in didactics, glottocodematics, psycholinguistics - in the specific manner of that discipline. Thus, different theories recommended for glottodidactic practice propose their own/differing didactic-methodological solutions. The point of departure that is the language to be taught is, however, constituted by linguistic theories, which set out the distinctive features of that language, and particular glottodidactic theories are concerned with the effective acquisition and mastery of these features.
\end{abstract}

KEYWORDS: theoretical discussions and practical implementation, "conceptual" thinking and formulation of expressions, glottodidactic processes, Pfeiffer's view: from practice to practice, three-phase glottodidactic actions.

SCHLÜSSELWORTE: theoretische Überlegungen und praktische Ausführungen, "begriffliches" Denken und Wort-Formulierungen, glottodidaktische Prozesse, Pfeiffers Auffassung: von Praxis zu Praxis, dreiphasiger Ablauf der glottodidaktischen Lehr-und Lern-Tätigkeiten. 
Homines, dum docent, discunt.

Die Menschen lernen, indem sie lehren.

Seneca, Epistulae morales ad Lucinum, 7, (17), 8.

\section{VORBEMERKUNGEN}

Im vorliegenden Beitrag wird versucht, ausgewählte fremdsprachenunterrichtliche Fragen sowohl vom theoretischen als auch vom praktischen Standpunkt aus zu erörtern und diese Betrachtungsweise auf eine gemeinsame und übergeordnete Unterrichtsgrundlage zu bringen, wo fremdsprachliche Lehr- und Lern-Prozesse unter institutionalisierten Be-dingungen stattfinden.

\section{THEORETISCHE GRUNDLAGEN DES FREMDSPRACHENUNTERRICHTS}

Eines der Hauptziele der theoretischen Betrachtungen bezüglich des Fremdsprachenunterrichts besteht darin, einerseits die den glottodidaktischen Prozessen zugrunde liegenden Gesetzmäßigkeiten zu erkennen, und andererseits auf die formulierten Gesetze, die den Ablauf dieser Prozesse bestimmen, hinzuweisen und diese darzustellen.

Gegenstand des glottodidaktischen Interesses sind, so kann man jedenfalls annehmen, erstens die Theorie, die zur konstruktiven Explizierung der wissenschaftlich-linguistischen Forschungsergebnisse beiträgt, und zweitens die (Unterrichts-)Praxis, in die die gewonnenen theoretischen Resultate übertragen und in der sie möglichst optima forma angewandt werden. Auf diese Weise wird die Diskussion über die oft angesprochene Diskrepanz zwischen Theorie und Praxis anders verlaufen und deren Ergebnisse werden im Sinne einer bestmöglichen Auswertung auch anerkannt und akzeptiert werden. Oder anders gesagt: Es geht um Relationen zwischen den theoretischen Überlegungen, in denen wissenschaftlich begründete Konzepte zur Erklärung der fremdsprachlichen Lehr- und Lern-Prozesse oder deren Phasen ausgearbeitet werden, und den praktischen Lehr- und Lern-Handlungen, die in bestimmten Unterrichtseinheiten realisiert werden.

Da jeder Fremdsprachenunterricht auf entsprechend aufbereiteten Materialien basiert, können nicht nur die Theoretiker zur Erarbeitung der sprachlichen Grundlagen für die Lehr- und Lern-Prozesse einen bedeutenden Beitrag liefern, sondern auch die Praktiker, die die glottodidaktischen Lehrprozesse im Klassenraum direkt organisieren und verwirklichen sowie die 
Ergebnisse der Lernprozesse beobachten, kontrollieren und - im Bedarfsfall - auch korrigieren.

Dementsprechend darf man wohl behaupten, dass durch die theoretischfremdsprachlichen Forschungserkenntnisse nicht nur der Ablauf der Lehr- und Lern-Prozesse analysiert und erklärt werden kann, sondern auch bestimmte Konzepte ausgearbeitet und vorgelegt werden können, die für die praktischen Ausführungen anregende Hinweise liefern.

Wesentlich für die Wissenschaft sind - nach der Sentenz Non progredi est regredi - theoretische Grundlagenforschungen in bestimmten Bereichen der Wirklichkeit. Eines der wissenschaftlichen Untersuchungsgebiete sind selbstverständlich die fremdsprachlichen Erlernungs- und Aneignungsprozesse. Die sukzessive Erlernung der fremdsprachlichen Strukturen und deren rezeptive und produktive Dynamisierungen in den Kommunikationssituationen führen des Weiteren zu deren Aneignung, was dem Lernenden erlaubt, als selbstständiger Kommunikationspartner zu fungieren.

Bei theoretischen Überlegungen eines fremdsprachenunterrichtlichen Bereiches sowie bei anderen wissenschaftlich-theoretischen Betrachtungen hat man es wahrscheinlich mit gedanklichen Vorstellungen vom gemeinten Sachverhalt zu tun, der im Bewusstsein des Forschenden auf solch eine Art und Weise begrifflich abgebildet wird, dass ihm eine bestimmte semantische Bezeichnung, ein konkretes Wort zugeordnet wird.

Dementsprechend führt der bereits umrissene kognitiv-sprachliche Weg vom sog. "begrifflichen“ Denken zu Wort-Formulierungen. Dieser Prozess trägt dazu bei, dass sich auf Grund solcher Operationen auch Neuschöpfungen (z.B. Glottodidaktik) mit eigentümlichen, d.h. mit spezifischen für das Wesen der kennengelernten Wirklichkeitsobjekte, Bedeutungen herausbilden, die als bestimmte Termini in den Wissenschaftskreisen anerkannt und akzeptiert werden.

Der Fremdsprachenunterricht ist durch seinen vielfältig aneinandergebundenen Ablauf als Verknüpfung von bestimmten Hauptprozessen gekennzeichnet, d.h. von Lehrprozess, Übertragungsprozess der Informationsstrukturen, Lernprozess, die sich weiter unter bestimmten Aspekten in einzelne Subprozesse einteilen lassen. Aber die Interpretation jedes einzelnen Prozesses lässt in vielen Wissenschaftsdisziplinen für sich typische semantische Erklärungsmöglichkeiten $\mathrm{zu}$, und so wird die Bedeutungserschließung des Wortschatzes in der Didaktik, der Kodematik und der Psycholinguistik nicht auf die gleiche Weise prozessual begriffen und dargestellt. Um das Wesen der Prozesse genauer zu begreifen, empfiehlt es sich, eine wissenschaftlich-multidisziplinäre und -interdisziplinäre Zusammenarbeit auf dem Gebiet des Fremdsprachenunterrichts aufzunehmen und im 
Weiteren konstant fortzusetzen. Die durch multidisziplinäre und interdisziplinäre Überlegungen gewonnenen Erkenntnisse bringen einerseits neue Einsichten in das Wesen der fremdsprachenunterrichtlichen Prozesse und deren Eigenschaften mit sich, andererseits auch neue Wörter, die für die Praxis relevant sind und selbstverständlich begrifflicher Beherrschung bedürfen. Hier einige Beispiele, die die fremdsprachenunterrichtlichen Handlungen als spezifische Kommunikationsvorgänge $\mathrm{zu}$ betrachten erlauben (vgl. ausführlicher Zabrocki 1966 u. 1975): (Glotto-)Kodematik - Kode, (De-)Kodierung), Kybernetik - Sender, Empfänger, Rückkoppelung, Steuerung, Psycholinguistik - Internalisierung, Externalisierung.

\section{ZUR UMSETZUNG DER THEORIE IN DIE PRAXIS}

In den praktischen Ausführungen des Fremdsprachenunterrichts lassen sich im Allgemeinen zwei Komplexfragen hervorheben:

1. Die nacheinander folgenden praktischen Tätigkeiten des Lehrenden, der als Sender das zu lernende fremdsprachliche Material den Empfängern (den Lernenden) darbietet, erklärt und deren Erfolge auch kontrolliert.

2. Die ganzheitliche Dekodierung der empfangenen Informationssignale und -strukturen durch die Lernenden, d.h. das richtige Verstehen der entschlüsselten Strukturen und deren Aufbewahrung für eine gewisse Zeit im Gedächtnis.

Der Lehrende wird oft als erfahrener Praktiker betrachtet, der verpflichtet ist, das im Lehr-Lern-Buch für den Unterricht aufbereitete fremdsprachliche Material schrittweise so darzubieten, dass dessen einzelne lexikalische und grammatische Strukturen von den Lernenden erkannt und erlernt werden können.

Beim Ausführen der betreffenden Tätigkeit stützt sich der Lehrende einerseits auf die linguale und die linguistische Ausbildung und andererseits auf die im Studium erworbenen glottodidaktischen (Fach-)Kenntnisse, die für die Lehr- und Lern-Prozesse unabdingbar sind.

$\mathrm{Zu}$ den bereits genannten Ausbildungs- und Tätigkeitseigenschaften kommen noch die neuen Theorien über die - beispielsweise - kodematischen oder psycholinguistischen Grundlagen des Fremdsprachenunterrichts hinzu, die dessen Prozesse unter spezifischen Aspekten betrachten. Auf solch eine Art und Weise werden Theorien aufgestellt, die mit bestimmten Termini begrifflich erläutert werden. Die Umsetzung der Theorien in die 
Praxis setzt fachliches Wissen und eine fachlich-glottodidaktische Kompetenz seitens der Lehrenden voraus.

Die Relationen zwischen der Theorie und dem Praxisbezug lassen sich sowohl in den Lehrprozessen, in denen das fremdsprachliche Material präsentiert und erklärt wird, als auch in den Lernprozessen verwirklichen, in denen die einzelnen Strukturen des Materials richtig entschlüsselt und stufenweise angeeignet werden. Die Darbietung des fremdsprachlichen Materials durch den Lehrenden (= primärer Sender) erfordert auch eine suprasegmentale oder eine außersprachliche Steuerung auf der interindividuellen Übertragungsebene, damit die Lernenden (= primäre Empfänger) die aufgenommenen Informationssignale ganzheitlich dekodieren und in Form von Sprachstrukturmatrizen im Gedächtnis speichern können.

Ein weiteres praxisbezogenes Problem betrifft die Reproduktions- und Produktionsmöglichkeiten der für die Kommunikation benötigten Informationsstrukturen durch den Lernenden (= sekundärer Sender), der im Rückkoppelungsgefüge mit dem Lehrenden (= sekundärer Empfänger) fungiert. In diesem Fall übernimmt der Lehrende auch die Kontrolle und bei Bedarf die Korrektur der Lern- und Kommunikationsleistung seines Unterrichtsteilnehmers.

Die aufgestellten Theorien, die vorgeschlagen werden, sind durch ihre Vielfalt gekennzeichnet, die zusätzlich eine didaktisch-methodische Vielfalt aufweisen, weil die Persönlichkeiten der Lehrenden nicht identisch sind, d.h. jeder Lehrende besitzt seine individuelle linguistische und didaktischmethodische Kompetenz, die sich in jeder Unterrichtseinheit manifestiert. Jede Theorie, die für die praktische Anwendung von Nutzen ist, erfordert aber auch eine Anpassung an bestimmte Strukturen, die unter glottodidaktischen Bedingungen gelehrt und gelernt werden (vgl. dazu Helbig 1994: 201). Anders gesagt: die Theorie bedarf sowohl einer fachlichen Verarbeitung als auch einer didaktisch-methodischen Umsetzung im und für den Fremdsprachenunterricht.

Ähnliche Probleme in der Anwendung der Theorie zu bestimmten praktischen Zwecken tauchen auf dem Gebiet der linguistischen Grammatik und der didaktischen Grammatik auf. Die linguistische Grammatik versteht sich - so Jung (1975: 7 u. 33) - als ein theoretisches Konstrukt, dass nicht direkt auf die Applikabilität hinweist, die didaktische Grammatik dagegen bezieht sich auf die Praxis des Fremdsprachenunterrichts, und deshalb wird sie nur solche praxisrelevanten Konzeptionen und Methoden darlegen, die den Lernbedingungen entsprechen und durch die Lernenden umgesetzt werden können (Edmondson 1982: 40). Auf diese Weise werden theoretisch ausgearbeitete Konstrukte als lehr- und lernbezogene Vorschläge und Realisie- 
rungsmöglichkeiten im Fremdsprachenunterricht verstanden. Schließlich muss Folgendes unterstrichen werden: Theoretisch fundierte Verfahren machen es zwingend notwendig, dass man zugleich über deren didaktischmethodische Umsetzung reflektiert und versucht, sie in der Unterrichtpraxis $\mathrm{zu}$ verwirklichen.

Mit praktischen Fragen des Fremdsprachenunterrichts beschäftigt sich Pfeiffer in seiner Monographie ${ }^{1}$, wo wesentliche Konzepte und Vorschläge für die Lehr- und Lern-Prozesse ausführlich erläutert werden. Obwohl der zweite Teil des Monographie-Titels den fremdsprachlichen Unterrichtsweg von Praxis zu Praxis unterstreicht, legt der Autor ebenso großen Wert auf die theoretischen Kenntnisse.

Die Ziele, die in dem ersten Kapitel (S. 13-31) verfolgt werden, dienen insbesondere den wissenschaftlich-theoretischen Fragen und zeigen die theoretischen Grundlagen der Glottodidaktik auf. Dies bedeutet, dass die Diskussion über fremdsprachenunterrichtliche Probleme ebenso die Theorie eröffnet. Pfeiffer (2001: 20f.) stellt auf der Grundlage der Modelle des glottodidaktischen Gefüges von Grucza (1978: 8) und Woźniewicz (1987: 81) sein eigenes Modell dar, das auch die glottodidaktischen Materialien, die Lehrund Lern-Methoden, die Unterrichtsbedingungen und die objektive Wirklichkeit umfasst.

Praktisch zu lösende Aufgaben sind Überlegungsgegenstand sensu largo des zweiten Kapitels, in dem zunächst drei prädidaktische Programmierungsphasen der glottodidaktischen Prozesse unterschieden werden, von denen die wissenschaftlich-theoretischen Prozesse exponiert sind (S. 32 ff.). Der Wissenschaftlichkeit und ihrer Relevanz für die didaktisch-methodische Arbeit des Lehrenden im Fremdsprachenunterricht wird besondere Aufmerksamkeit geschenkt (s. S. 94 u. 138). Praktisch-fremdsprachenunterrichtliche Ausführungen enthalten in den weiteren Kapiteln genaue und gut ausgearbeitete didaktisch-methodische Konzepte und Vorschläge, die folgende Probleme betreffen: methodische Grundlagen des Fremdsprachenunterrichts, Faktoren der effektiven Unterrichtspraxis, Lehrprogramme, Präparation und Evaluation der fremdsprachlichen Materialien, allgemeine Ausbildung der Lehrenden und ihre Tätigkeiten. Aus den allgemeinen Sachformulierungen ergeben sich Aufgaben, Anregungen und Empfehlungen zu den praktischen Ausführungen sowohl für die Lehrenden als auch für die Lernenden.

Im Hinblick auf die von Pfeiffer diskutierten Relationen zwischen Theorie und Praxis im Fremdsprachenunterricht lassen sich deren Verbindungen folgendermaßen konzipieren:

\footnotetext{
${ }^{1}$ Es handelt sich um folgende Monographie: Pfeiffer 2001.
} 


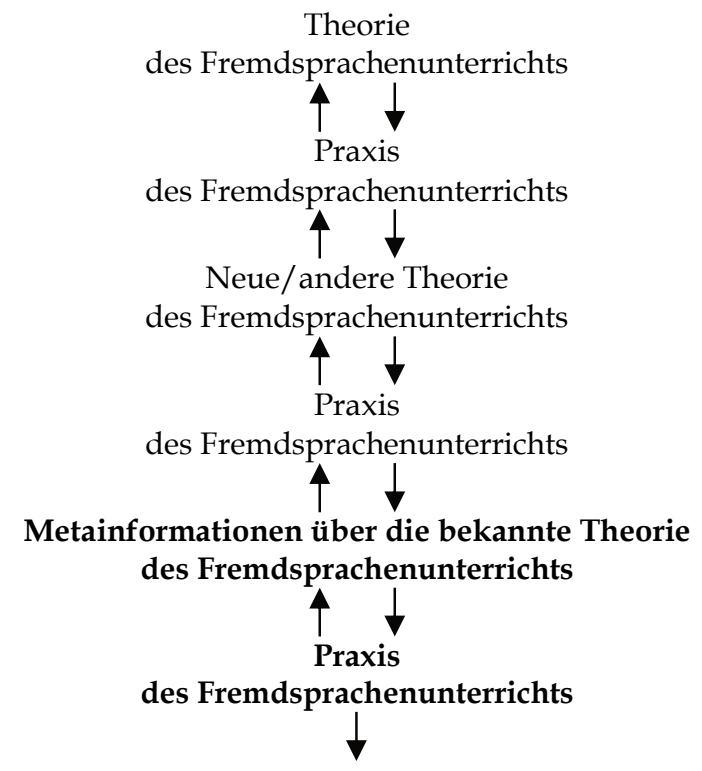

Abbildung 1. Mehrstufige Verbindungsverhältnisse zwischen Theorie und Praxis im Fremdsprachenunterricht

Aus den oben geschilderten fremdsprachenunterrichtlichen Fragen und den dargestellten Relationen (Abb. 1.) ergeben sich folgende Konstatierungen: Die Theorie liefert der Praxis nützliche und konstruktive Informationen, dementsprechend wendet die Praxis diese Informationen zweckmäßig an. Die Praxis inspiriert auch zum Neuüberdenken und trägt zur Weiterentwicklung der theoretischen Kenntnisse bei. Die Verbesserung der fremdsprachlichen Ergebnisse beruht auf den wechselseitigen Betrachtungsverhältnissen zwischen Theorie und Praxis. Mit anderen Worten - es geht darum, dass jeder Unterrichtspraxis eine bestimmte theoretische Basis zugrunde liegt, die in den praktischen Ausführungen - so Kerkhoff (1983: 58) konkretisiert wird.

Dass die fremdsprachliche Unterrichtspraxis nicht von den linguistischen Forschungen zu trennen ist, erklärt zutreffend Lewandowski (1982: 37), der ebenfalls die Theoriebildung in einem engen Zusammenhang mit der Analyse der realen Lehr- und Lern-Situationen sieht.

In den theoretischen Überlegungen werden einerseits die linguistischen Theorien der Einzelsprachen behandelt, andererseits die glottodidaktischen Theorien, die sich mit den Fragen der Erlernung und der Aneignung einer bestimmten Sprache beschäftigen. In unserem Falle ist es Deutsch als Fremdsprache, deren einzelne Strukturen durch spezifische linguistische 
Eigenschaften gekennzeichnet sind und dementsprechend spezielle Lehrund Lern-Methoden erfordern.

Die Verzahnung zwischen den theoretisch-glottodidaktischen Überlegungen und den praktisch-glottodidaktischen Ausführungen lässt sich - unseres Erachtens - in einem dreiphasigen Prozess darstellen, der schematisch wie folgt konzipiert wird:
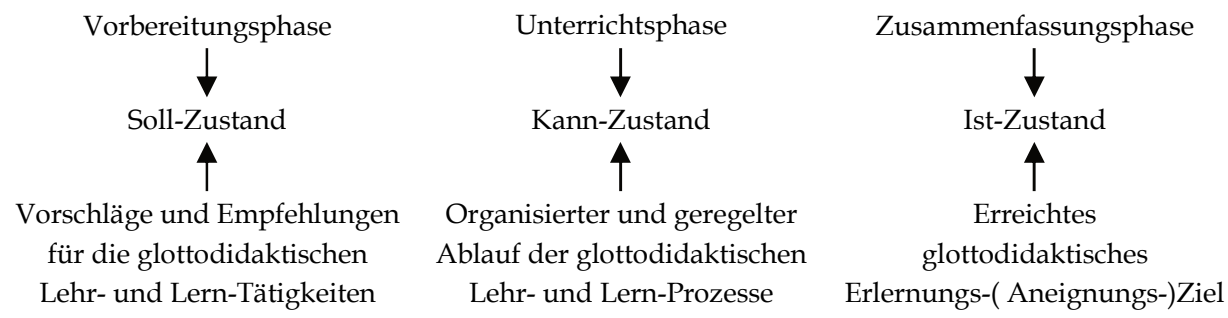

Abbildung 2. Glottodidaktische Phasen und ihre kennzeichnenden Zustände

Die Vorbereitungsphase, die einen präglottodidaktischen Charakter hat, befasst sich mit den zu lehrenden und zu lernenden neuen sowie auch mit denjenigen Strukturen, die schon erlernt wurden aber zusätzlicher Wiederholung sowie fester Einprägung bedürfen. Um die geplanten Lehr- und Lern-Ergebnisse erzielen zu können, werden auch solche theoretischen Kenntnisse berücksichtigt, welche den Lehr- und Lern-Vorgang erfolgreich steuern (Näheres hierzu Szczodrowski 2001: 47-97). Dementsprechend werden drei folgende Fragen hervorgehoben: Was, Wie und Wem, wobei sich die erste und zweite Frage auf das zu lehrende Material und dessen didaktisch-methodische Darstellung beziehen, die dritte dagegen auf den Bewältigungsgrad der Fremdsprache durch den Lernenden abzielt.

Kennzeichnend für die Unterrichtsphase sind die sich als status quo vollziehenden Lehr- und Lern-Prozesse, unter denen sich zwei Etappen unterscheiden lassen. Zum Ersten wird das bekannte Material auf verschiedene Art und Weise wiederholt, um es zu festigen. Empfohlen werden mündliche Interaktionen, deren Teilnehmer die Lernenden als fungierende Sender und Empfänger sind. Der vorgeschlagene Kann-Zustand bedeutet, dass es verschiedenartige Möglichkeiten gibt, durch die das Gelernte von den Lernenden wiederholt und tiefer eingeprägt wird. Als Beispiele gelten hier: fremdsprachige Beantwortung von Fragen nach Fragen, kurze Wiedergabe des Textes, Bildbeschreibungen, dialogisches Sprechen in vorgestellten oder simulierten Situationen u.v.a.m. Zum Zweiten geht es um die Darbietung und die Erklärung des neuen Materials sowie dessen Erlernungskontrolle durch den 
Lehrenden. Diese Tätigkeiten bezüglich der Organisation und der Ordnung des Unterrichts sind die Domäne des Lehrenden, dem ebenfalls mehrere didaktisch-methodische Mittel und Wege zur Verfügung stehen.

In der Zusammenfassung (postglottodidaktische Phase) werden vom Lehrenden sowohl der quantitative als auch der qualitative Ist-Zustand der erlernten und angeeigneten Strukturen beurteilt. Welche Mittel der Lehrende für die Kontrolle der durch die Lernenden erzielten Leistungen anwendet (mündliche oder schriftliche), hängt von ihm ab. Im Allgemeinen handelt es sich darum, den Ist-Zustand der gelernten und der angeeigneten Strukturen zu erkennen und ebenso zu reflektieren, wie man den Unterricht theoretisch und praktisch optimieren könnte.

\section{SCHLUSSWORT: THEORIA CUM PRAXI}

Wie anfangs angedeutet, wurden in unserem Beitrag ausgewählte theoretische und praxisorientierte Probleme des Fremdsprachenunterrichts angeschnitten und besprochen. Diese abschließenden Bemerkungen sollen noch einmal verdeutlichen, dass theoretische Konzepte und Vorschläge mit den praktischen Ausführungen auf dem Gebiet des neusprachlichen Unterrichts übereinstimmen sollen.

Die dargelegten Ausführungen sollen einen Beitrag dazu leisten, dass der Graben zwischen Theorie und Praxis im Fremdsprachenunterricht zu überbrücken ist. Dies bedeutet auch, praxisbezogene Theorien mit dem Ziel $\mathrm{zu}$ entwickeln, den neusprachlichen Unterricht so effektiv wie möglich zu gestalten.

\section{LITERATURVERZEICHNIS}

Edmondson, W. J. (1982). Die didaktische Grammatik und ihre Relevanz für die Linguistik. In: W. Kühlwein / A. Raasch (Hrsg.), Stil: Komponenten - Wirkungen. Band II (S. 40-43). Tübingen: Gunter Narr Verlag.

Grucza, F. (1978). Glottodydaktyka w świetle modeli komunikacji językowej. In: F. Grucza (Hrsg.), Teoria komunikacji językowej a glottodydaktyka (S. 7-26). Warszawa: Uniwersytet Warszawski.

Helbig, G. (1994). Das Verhältnis von Sprachwissenschaft und Sprachunterricht im Wandel der Zeiten. Deutsch als Fremdsprache, 4, 201-208.

Jung, L. 1975. Linguistische Grammatik und Didaktische Grammatik. Frankfurt a. M., Berlin.

Kerkhoff, L. van den (1983). Zum Verhältnis von Sprachtheorie und Sprachdidaktik. Frankfurt a.M., Bern, New York: Peter Lang.

Lewandowski, Th. (1982). Das Verhältnis von theoretischer und angewandter Linguistik. Sprachdidaktik und didaktische Grammatik. In: W. Kühlwein / A. Raasch, (Hrsg.), Stil: Komponenten - Wirkungen. Band II (S. 36-39). Tübingen: Gunter Narr Verlag. 
Pfeiffer, W. (1975). Teoretyczne podstawy preparacji materiałów glottodydaktycznych. Poznań: Uniwersytet im. Adama Mickiewicza w Poznaniu.

Pfeiffer, W. (2001). Nauka języków obcych. Od praktyki do praktyki. Poznań: Wagros.

Szczodrowski, M. (2001). Steuerung fremdsprachlicher Kommunikation. Gdańsk: Wydawnictwo Uniwersytetu Gdańskiego.

Woźniewicz, W. (1987). Kierowanie procesem glottodydaktycznym. Warszawa: PWN.

Zabrocki, L. (1966). Kodematische Grundlagen der Theorie des Fremdsprachenunterrichts. Glottodidactica. An International Journal of Applied Linguistics, 1, 3-42.

Zabrocki, L. (1975). Kybernetische Modelle der sprachlichen Kommunikation. Wrocław, Warszawa, Kraków, Gdańsk: Ossolineum.

Received: 18.04.2018; revised: 7.08.2018 